\title{
Tafonomía actualística de huesos de anfibios predados por estrigiformes del centro de Argentina. Contribuciones para estudios paleontológicos y arqueológicos
}

\author{
Julián MIGNINO
}

FONCyT, IDACOR-CONICET, Museo de Antropología, Facultad de Filosofía y Humanidades, Universidad Nacional de Córdoba, Av. Hipólito Yrigoyen 174, CP 5000. Córdoba, Argentina. julianmignino@ffyh.unc.edu.ar

Mignino, J. 2021. Tafonomía actualística de huesos de anfibios predados por estrigiformes del centro de Argentina. Contribuciones para estudios paleontológicos y arqueológicos. [Actualistic taphonomy of amphibian bones predated by strigiforms from central Argentina. Contributions for palaeontological and archaeological studies]. Spanish Journal of Palaeontology, 36 (1).

\section{RESUMEN}

Se presenta un análisis tafonómico comparativo de restos de anfibios recuperados en egagrópilas de aves rapaces estrigifomes provenientes del centro de Argentina, en la provincia de Córdoba: Tyto furcata, Megascops choliba y Athene cunicularia. El estudio se basó en el promedio de abundancia relativa de elementos anatómicos, índices de representatividad de partes esqueletales y se evaluó fracturación y digestión. Los resultados derivados del análisis de representatividad y abundancia anatómica revelaron promedios elevados de abundancia relativa en todos los casos. Los grados de fractura y digestión fueron evaluados como leves para $T$. furcata, leves-moderados para M. choliba y moderados para $A$. cunicularia. En general, estas evaluaciones coinciden con las referencias previas. Sin embargo, se detectaron variaciones en los patrones de fracturación y digestión. Los datos presentados aportan información como análogos modernos para evaluar la presencia de esta clase de restos en contextos arqueológicos y paleontológicos.

Palabras clave: Microvertebrados; Tafonomía; Análogos Modernos; Depredadores Sudamericanos.

\begin{abstract}
A comparative taphonomic analysis of amphibian remains recovered from Strigiforms raptors pellets from central Argentina in the Córdoba province is presented: Tyto furcata, Megascops choliba and Athene cunicularia. The results derived from the analysis of the representativeness and anatomical abundance revealed high percentages of relative abundance in all cases. The degrees of breakage and digestion were evaluated as light for $T$. furcata, light-moderate for $M$. choliba and moderate for A. cunicularia. In general, these evaluations are consistent with previous references. However, variations in breakage and digestion patterns were detected. Data presented provide information as modern analogs to assess the presence of this class of remains in archaeological and palaeontological contexts.
\end{abstract}

Keywords: Microvertebrates; Taphonomy; Modern analogs; South American Predators. 


\section{INTRODUCCIÓN Y OBJETIVOS}

La presencia de anfibios ha sido documentada en distintos contextos arqueológicos y paleontológicos de todo el mundo (Pinto-Llona \& Andrews, 1999; Blain et al., 2008; Stoetzel et al., 2012; Alcaraz, 2017). Su aparición ha sido atribuida a una serie de hipótesis que enfrentan variables de índole cultural y natural, como entrampamientos naturales, mortalidad durante épocas de hibernación, estivación, depredación de aves rapaces y mamíferos carnívoros, consumo humano y actividades rituales (e.g., Andrews, 1990; Furts, 1994; Shultes \& Hofmann, 2000; Hüster, 2004; Kyselý, 2008).

Desde esta perspectiva, la información generada a partir de la evaluación y construcción de referentes tafonómicos se ha constituido como una herramienta imprescindible a la hora de conocer y comprender los agentes y factores involucrados en la presencia de esta clase de registros (Andrews, 1990), permitiendo discriminar distintas variables derivadas de procesos diagenéticos y bioestratinómicos.

Tal como se mencionó, la intervención de aves rapaces en la acumulación de microvertebrados ha sido documentada en numerosos casos. A la vez, los estudios tafonómicos y taxonómicos de presas contenidas en sus egagrópilas se han constituido como un proxydata ambiental útil a la hora de realizar inferencias paleobiológicas y paleoecológicas (Andrews, 1990; Pardiñas, 1999; Stoessel et al., 2008; Fernández et al., 2012; Muzzopappa et al., 2020).

En Argentina, se ha evidenciado un marcado incremento de publicaciones y espacios específicos de la temática, que generaron marcos de referencia y desarrollo de datos locales como análogos modernos de mayor precisión (Fernández et al., 2017; Montalvo \& Fernández, 2019).

Sin embargo, si bien se ha dado un incremento notable de estudios de restos de microvertebrados, estos han sido enfocados, principalmente, en pequeños mamíferos y aves (Frontini \& Escosteguy, 2015; Montalvo \& Fernández, 2019). Otros taxones como peces y anfibios, posiblemente como producto de su menor representatividad dentro de las asociaciones faunísticas, pasaron a ocupar un segundo plano (Montalvo \& Tejerina, 2009; Alcaraz, 2017).

En este sentido, una de las causas de menor representación de anfibios en acumulaciones no humanas puede estar vinculada con los hábitos tróficos de los predadores. Un ejemplo de ello lo constituyen agentes productores como Tyto furcata, cuyo rol como depredador de roedores sigmodontinos en un $80 \%$ de la totalidad de su dieta es ampliamente conocido (Bellocq, 2000). Sin embargo, el comportamiento oportunista de esta clase de aves rapaces puede generar casos de elevada batracofagia, hecho que tensiona los modelos de dieta propuestos, a la vez que incluyen datos sobre implicancias ambientales interesantes (Fernández et al., 2009).
Por otro lado, si bien las investigaciones tafonómicamente orientadas de huesos de anfibios en contextos europeos y africanos han sido desarrolladas (Pinto-Llona \& Andrews, 1999; Blain et al., 2008; Stoetzel et al., 2012), los estudios actualísticos sobre acumulaciones por causas no antrópicas son aún escasos (Bisbal-Chinesta et al., 2020).

En correlación, Argentina presenta un vacío de información vinculado a las alteraciones que ejercen distintos predadores (aves rapaces y mamíferos carnívoros) sobre huesos de anfibios, y a la riqueza interpretativa que tienen esos datos para evaluar su presencia en distintos contextos.

Por ello, y con la intención de incrementar el grado de conocimiento sobre patrones de modificación ósea por parte de aves rapaces en restos de anfibios, se presenta un estudio tafonómico comparativo de huesos ingeridos y digeridos por búhos y lechuzas (Strigiformes) del centro de Argentina, en la provincia de Córdoba. Al respecto, el objetivo principal radicó en conocer características diagnósticas y aportar datos que permitan categorizar y evaluar su presencia como productores de restos óseos en contextos arqueológicos y paleontológicos.

\section{AGENTES PRODUCTORES}

Se analizaron tres conjuntos de egagrópilas de aves rapaces pertenecientes al orden estrigiformes. La primera de ellas corresponde a la lechuza de campanario (Tyto furcata), una rapaz de tamaño mediano (290-440 mm) y de distribución cosmopolita, con hábitos preferentemente nocturnos y crepusculares, que se alimenta mayormente de pequeños mamíferos (roedores, marsupiales y murciélagos), aves, anfibios y, ocasionalmente, insectos (Bellocq, 2000). Suele anidar en huecos de árboles, construcciones abandonadas, aleros rocosos y cualquier otra estructura que presente refugio y reparo.

Recientemente, Uva et al. (2018) basados en estudios filogenéticos, sugirieron que la lechuza de campanario se divide en tres unidades evolutivas. En este sentido, proponen tres especies diferentes: Tyto furcata en América; Tyto alba en Europa y África; y Tyto javanica en Asia y Oceanía.

Debido a la escasez de estudios tafonómicos derivados del análisis de huesos de anfibios se realizará una comparación entre la especie americana y la europea.

La lechuza de campanario es considerada uno de los agentes productores de asociaciones microvertebrados más frecuentes en sitios paleontológicos, arqueológicos y modernos (Andrews, 1990; Pokines \& Kerbis, 1997; Pardiñas, 1999; Fernández et al., 2011, 2012; Mignino et al., 2018), y fue categorizada en estudios tafonómicos como una rapaz que produce modificaciones mínimas sobre 
los huesos de sus presas (Andrews, 1990; Pinto-Llona \& Andrews, 1999; Montalvo \& Fernández, 2019).

Por otro lado, se evaluaron egagrópilas de Alicucu común (Megascops choliba), uno de los búhos neotropicales de tamaño pequeño (220-240 mm) más comunes de nuestro continente (Hilty \& Brown, 1996). Su distribución comprende desde las islas de Centroamérica hasta el centro de la Argentina y Uruguay, ocupando una variada cantidad de ambientes, entre los cuales se incluyen bosques abiertos, bordes de bosques, sabanas, áreas de cultivo y zonas urbanas (König \& Weick, 2010). Esta rapaz posee hábitos crepusculares y nocturnos, nidifica en huecos de árboles o nidos abandonados por otras aves, y las escasas investigaciones sobre su alimentación indican una dieta estacionalmente oportunista, que incluye mayormente insectos (e.g., grillos, mariposas nocturnas, coleóptero, arañas) en estaciones cálidas, con un incremento de pequeños vertebrados hacia épocas frías (e.g., anfibios, quirópteros y roedores) (del Hoyo et al., 1999; Delgado, 2007). Aunque es conocido su rol como depredador de microvertebrados, el estudio tafonómico de las modificaciones que produce sobre huesos es aún desconocido (Montalvo \& Fernández, 2019).

Finalmente, se analizaron restos producidos por la lechucita vizcachera (Athene cunicularia), una rapaz de tamaño pequeño (190-250 mm) con una amplia distribución en todo el Cono Sur sudamericano, que nidifica en cuevas y madrigueras abandonadas por otros animales de hábitos fosoriales y semi-fosoriales (König \& Weick, 2010). A. cunicularia posee hábitos preferentemente diurnos y su alimentación depende de las fluctuaciones estacionales en la disponibilidad de presas, por lo que se la considera como una rapaz oportunista (Montalvo et al., 2020).

Los estudios tafonómicos sobre fractura y digestión ubican a $A$. cunicularia en la categoría moderada de modificación, tanto en muestras de anfibios como de pequeños mamíferos (Montalvo \& Tejerina, 2009; Montalvo et al., 2020).

\section{3. ÁREAS DE ESTUDIO}

Las muestras estudiadas provienen de tres localidades de la provincia de Córdoba (Argentina) con distintas fisonomías, rangos altitudinales, niveles de precipitación, temperaturas, tipos de suelo, vegetación y grados de impacto antrópico (Cabido et al., 2018) (Fig. 1). Dos de ellas (T. furcata y A. cunicularia) provienen de Salinas Grandes (en adelante

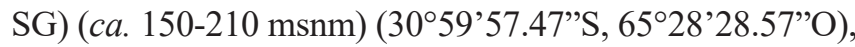
un área donde las lluvias están fuertemente restringidas a causa de cordones montañosos que actúan como barrera natural. Las precipitaciones acumuladas en estaciones frías y secas no superan los $80 \mathrm{~mm}$, mientras que, en estaciones cálidas y húmedas, el promedio supera los $400 \mathrm{~mm}$.
El estrato vegetal se caracteriza particularmente por la presencia de arbustales, en especial de jumes (Heterostachys ritteriana y Allenrolfea patagonica) y jarillas (Larrea divaricata y L. cuneifolia). En los suelos de mayor elevación con menor salinidad, se registra quebracho colorado santiagueño (Schinopsis quebracho-colorado) y el quebracho blanco (Aspidosperma quebracho-blanco) (Cabido et al., 2018).

El segundo de los conjuntos analizados ( $M$. choliba) proviene del valle de Ongamira ( $c$ a. 900-1500 msnm) (3046'28.94”'S, 64²4'49.57'O), en el Norte de las Sierras Chicas cordobesas. Este valle presenta un clima templado continental, en donde, en los meses de junio y julio, son ocasionales las nevadas por sobre los 1000 $\mathrm{msnm}$. Actualmente, se registran rangos de precipitaciones que van desde los $10 \mathrm{~mm}$ en estación seca (invierno) a los $150 \mathrm{~mm}$ en estación cálida y épocas de lluvia (verano). En este valle, el estrato arbóreo se encuentra dominado por la presencia del molle de beber (Lithraea molleoides), el orco quebracho (Schinopsis haenkeana), aguaribay (Schinus areira), coco (Zanthoxylum coco) y otras especies exóticas introducidas como diferentes tipos de pinos (Pinus spp.) y siempreverde (Ligustrum lucidum) (Robledo, 2019).

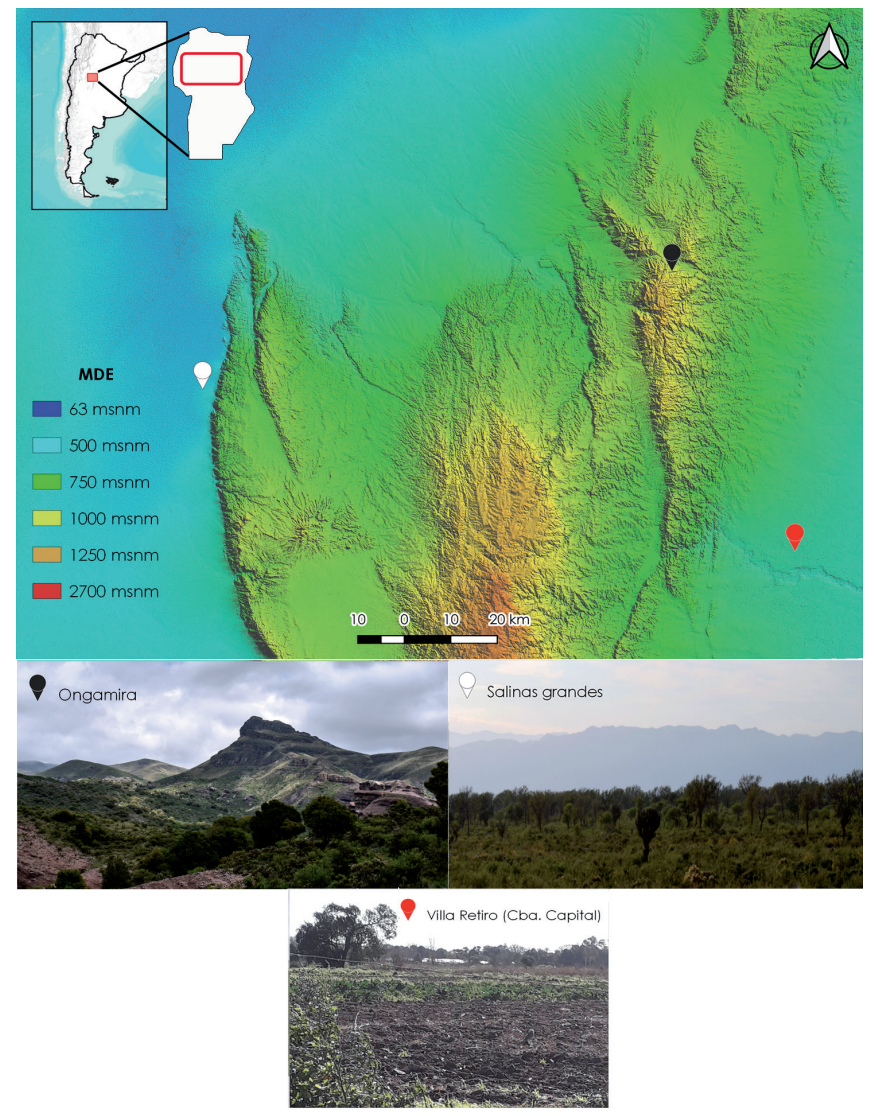

Figura 1. Localidades de estudio en la provincia de Córdoba (Argentina). Abreviaturas: MDE, Modelo de Elevación Digital; msnm, metros sobre el nivel del mar. 
Finalmente, se analizaron muestras (A. cunicularia) provenientes de Villa Retiro (VR, en adelante), en la capital provincial ( $c a .400-450 \mathrm{msnm}$ ) (31 $31^{\prime} 73.86^{\prime \prime} \mathrm{S}$, $64^{\circ} 14^{\prime} 59.45^{\prime \prime} \mathrm{O}$ ), un área donde las precipitaciones acumuladas alcanzan promedios de $6 \mathrm{~mm}$ en estaciones frías y secas y $128 \mathrm{~mm}$ en estaciones cálidas y húmedas. Este sector de muestreo se encuentra altamente modificado por agroecosistemas y emplazamientos urbanos caracterizados por la presencia de una variedad de pinos, siempreverde y fauna exótica.

\section{MATERIALES Y METODOLOGÍA}

Las muestras evaluadas corresponden a egagrópilas recolectadas entre septiembre y diciembre de 2019 en los distintos sectores de muestreo mencionados. Las mismas fueron desagregadas en seco utilizando instrumental quirúrgico. Se retiraron todos los restos de anfibios y se lavaron con agua, dejándolos secar al aire libre durante un día. Se calculó el número de especímenes identificados por taxón (NISP), el número mínimo de individuos (MNI) y el número mínimo de elementos (MNE). A fin de evaluar la preservación diferencial de elementos anatómicos, se calculó el índice de abundancia relativa de partes esqueletales (Andrews, 1990; Fernández-Jalvo \& Andrews, 1992). Este índice se expresa como: $\mathrm{Ri}=\mathrm{MNEi} /(\mathrm{Ei} *$ $\mathrm{MNI}) * 100$. Donde, $\mathrm{Ri}=$ abundancia relativa del elemento $\mathrm{i}, \mathrm{MNEi}=$ número de elementos i en la muestra, Ei = frecuencia de elementos $\mathrm{i}$ en el esqueleto y $\mathrm{MNI}=$ número mínimo de individuos.

Por otro lado, se calculó el índice que evalúa la representación de elementos craneanos y postcraneanos (fémur + húmero) / (mandíbula + maxilar) *100; y la representación de elementos esqueléticos del miembro anterior con respecto al posterior (húmero + radio-ulna) / (húmero + radio-ulna + fémur + tibio-fíbula) $* 100$ (Montalvo \& Tejerina, 2009, modificado de Bochensky et al., 2009). Finalmente, se evaluó la representatividad de elementos distales y proximales de los miembros (tibiofíbula + radio-ulna) / (fémur + húmero) $* 100$.

El estudio tafonómico de modificaciones sobre restos se basó en las directrices metodológicas propuestas por PintoLlona \& Andrews (1999). Las modificaciones causadas por fracturas y ácidos digestivos se evaluaron sobre la totalidad de los elementos anatómicos, aunque se hizo especial énfasis sobre el total de fémures, húmeros, tibio-fíbulas y radio-ulnas. Sobre estos, se analizó la acción digestiva y sus efectos, como astillamiento, redondeamiento, adelgazamiento, colapso y descamación. Las categorías empleadas para estudiar las modificaciones por digestión siguieron las pautas de Bisbal-Chinesta et al. (2020). Por ello, se clasificaron como leves (pequeños agujeros en articulaciones), moderadas (aumento de tamaño de orificios y exposición parcial de tejido esponjoso interno), y fuertes (pérdida parcial de región articular), y se incluyó la categoría extrema cuando existió pérdida total de región articular por corrosión. Mientras que, para el resto de los atributos derivados de la misma, se los clasificó en tres grados: sin modificación, leve y fuerte. Respecto de las fracturas, se clasificó de acuerdo a la pérdida de distintas secciones de los huesos. Desde aquellos elementos que se preservaron enteros (categoría 0), los que presentaron fragmentación intermedia (categoría 1, extremo proximal, categoría 2 , extremo distal y 3 , diáfisis completa) y aquellos que se recuperaron muy rotos (categoría 4, menos de la mitad de la diáfisis). Las observaciones tafonómicas e imágenes se obtuvieron a través de una Lupa Binocular Estereoscópica "Motic" SMZ-140-N2GG, con una cámara de 3.0 megapíxeles.

\section{RESULTADOS}

\subsection{Representación taxonómica y anatómica}

Se recuperaron un total de 992 elementos de anfibios (NISP) (Tabla 1). Los restos óseos recuperados en muestras de T. furcata y A. cunicularia de SG corresponden al género Leptodactylus, mientras que los ejemplares recuperados en conjuntos provenientes de Ongamira y VR fueron asignados específicamente a Rhinella arenarum (sapo argentino o sapo común).

Se obtuvo un MNE de 775 y un MNI de 42 en toda la muestra (ver Tabla 1). Sobre la base de esos datos, se calculó el promedio de abundancia relativa de partes esqueletales alcanzando un índice de $51,83 \%$ para $T$. furcata, 56,25\% para $A$. cunicularia (SG), 54,81\% para $M$. choliba y $57,36 \%$ para $A$. cunicularia de VR. En los restos producidos por T. furcata, predominaron tibio-fíbulas, iliones, fémures y mandíbulas; mientras que, en $A$. cunicularia, se preservaron mayoritariamente mandíbulas, húmeros y fémures. En muestras de $M$. choliba, predominaron tibio-fíbulas, húmeros, fémures e iliones. Finalmente, en el conjunto producido por $A$. cunicularia, proveniente de VR, prevalecieron en mayor porcentaje tibio-fíbulas, húmeros y fémures (Fig. 2).

El índice que evalúa la representatividad de elementos craneanos y postcraneanos indicó mayor representación de los segundos por sobre los primeros en los conjuntos provenientes de SG y Ongamira. Mientras que, en las muestras provenientes de A. cunicularia de VR, se registró mejor representación de elementos del cráneo.

El índice que evaluó la representatividad de elementos esqueléticos del miembro anterior respecto al posterior 


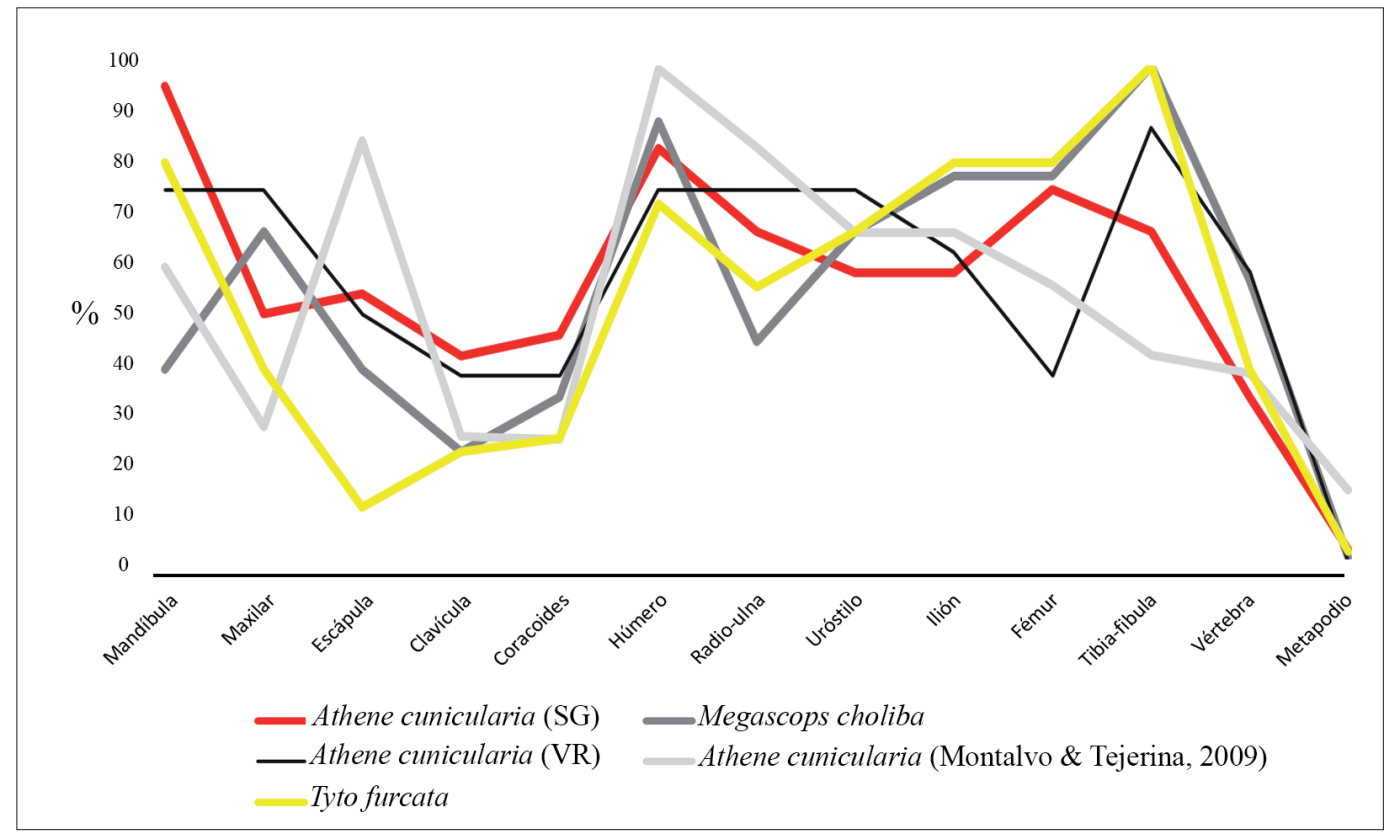

Figura 2. Comparación de abundancia relativa de elementos esqueletales de anfibios contenidos en egagrópilas de Tyto furcata, Megascops choliba y Athene cunicularia.

indicó, en todos los casos, mejor representación de los segundos.

Por otra parte, la relación entre elementos distales y proximales de los miembros indicó proporciones más equilibradas en las muestras de T. furcata, una mejor representación de los primeros por sobre los segundos en muestras de $A$. cunicularia de SG y $M$. choliba, y una leve tendencia a favor de elementos proximales en muestras de A. cunicularia de VR (ver Tabla 1).

Tabla 1. Valores de variables tafonómicas evaluadas en huesos de anfibios contenidos en egagrópilas de Tyto furcata, Megascops choliba y Athene cunicularia. Abreviaturas: número mínimo de elementos (MNE); número mínimo de individuos (MNI); porcentaje de abundancia relativa (Ri); postcráneo (pc); cráneo (c); húmero (h); radio (r); fémur (f); tibia (t).

\begin{tabular}{|c|c|c|c|c|}
\hline Predador & Tyto furcata & Athene cunicularia & Megascops choliba & Athene cunicularia \\
\hline Localidad & Salinas Grandes & & Ongamira & Villa Retiro \\
\hline Ubicación & $\begin{array}{l}30^{\circ} 59^{\prime} 57.47 ” \mathrm{\prime}, \\
65^{\circ} 28^{\prime} 28.57^{\prime \prime} \mathrm{O}\end{array}$ & & $\begin{array}{l}30^{\circ} 46^{\prime} 28.94^{\prime \prime} \mathrm{S} \\
64^{\circ} 24^{\prime} 49.57^{\prime \prime} \mathrm{O}\end{array}$ & $\begin{array}{l}31^{\circ} 31^{\prime} 73.86^{\prime \prime} \mathrm{S} \\
64^{\circ} 14^{\prime} 59.45^{\prime \prime} \mathrm{O}\end{array}$ \\
\hline msnm & $150-210$ & & $900-1500$ & $400-450$ \\
\hline Período de recolección & diciembre 2019 & & septiembre 2019 & \\
\hline $\mathrm{N}^{\circ}$ de egagrópilas analizadas & 56 & 40 & 73 & 6 \\
\hline$\%$ presencia de anfibios en egagrópilas & 17,85 & 47,5 & 31,5 & 100 \\
\hline Presas identificadas (tamaño) & Leptodactylus sp. & & Rhinella arenarum $(88-1$ & \\
\hline MNE & 313 & 223 & 166 & 73 \\
\hline MNI & 18 & 11 & 9 & 4 \\
\hline $\mathrm{Ri}$ & 51,83 & 56,25 & 54,81 & 57,36 \\
\hline Índice $\mathrm{pc} / \mathrm{c}$ & 127,9 & 108,5 & 157,8 & 75 \\
\hline Índice $(\mathrm{h}+\mathrm{r}) /(\mathrm{h}+\mathrm{r}+\mathrm{f}+\mathrm{t}) * 100$ & 41,14 & 51,42 & 42,8 & 54,5 \\
\hline Índice $(\mathrm{t}+\mathrm{r}) /(\mathrm{f}+\mathrm{h}) * 100$ & 101,8 & 84,2 & 86,6 & 108,3 \\
\hline$\%$ digestión & 55 & 67,14 & 75 & 72,72 \\
\hline$\%$ restos fracturados & 23,42 & 56,50 & 42,85 & 90,9 \\
\hline
\end{tabular}




\subsection{Fragmentación de los restos}

Los grados y porcentajes de fracturas analizados sobre elementos del postcráneo se presentan en la Tabla 2. Los restos digeridos por $T$. furcata evidenciaron porcentajes bajos de fracturación. Tal es así que húmeros y radioulnas se recuperaron completos en su totalidad. Mientras, fémures y tibio-fíbulas presentaron un grado mínimo de fractura. En menores proporciones, las muestras de $M$. choliba evidenciaron valores que superaron el $50 \%$ de completitud, exceptuando los registrados en tibio-fíbulas. Las muestras generadas por $A$. cunicularia, provenientes de ambas localidades, presentaron grados de fractura más severas, aunque los conjuntos provenientes de SG se conservaron con mayor nivel de completitud. Tal es así que se recuperaron más del $50 \%$ de húmeros y radioulnas sin fracturas.

\subsection{Digestión}

Se registraron distintos grados de alteración por digestión en todos los elementos anatómicos evaluados (Figs. 3 y 4). Alrededor del $50 \%$ de los restos proporcionados por $T$. furcata evidenciaron digestión, agrupando la mayoría de los ejemplares en la categoría leve de modificación. En menores proporciones, se registraron elementos asociados a la categoría moderada. En un solo caso (tibio-fíbula) se observaron alteraciones de tipo fuertes.
En tanto, las muestras acumuladas por M. choliba exhibieron acción digestiva en un $75 \%$ de los elementos, asociada principalmente con la categoría leve. En menor porcentaje se destacaron húmeros y tibio-fíbulas con modificaciones de tipo moderadas y fuertes.

En contraposición, las alteraciones más severas se observaron en ambas muestras acumuladas por $A$. cunicularia. Los valores de digestión fueron similares a los obtenidos en muestras de M. choliba. Sin embargo, se concentraron principalmente en las categorías leve y moderada. Mientras que, en menor proporción, se registraron modificaciones de tipo fuerte $\mathrm{y}$, en el caso de los húmeros, de grado extremo.

Otras variables asociadas a la digestión fueron evaluadas sobre la totalidad de los huesos (Figs. 4 y 5). Se destaca que, en muchos casos, se registraron distintos atributos en un mismo elemento anatómico. En primera instancia, los efectos del redondeamiento se observaron en mayores proporciones sobre huesos digeridos por A. cunicularia en ambas localidades ( $c a .40 \%$ ), mientras que, en los elementos digeridos por T. furcata y M. choliba, las proporciones disminuyeron considerablemente (ca. 3-11 $\%)$. En todos los casos, los efectos del redondeamiento fueron de tipo leve. Los astillamientos paralelos a la estructura fibrosa de los huesos indicaron proporciones similares, destacándose en restos acumulados por $A$. cunicularia (ca. 39-45 \%), mientras que, para T. furcata y M. choliba, los valores no superaron el $5 \%$. Para este

Tabla 2. Proporción y categorías de fractura de elementos postcraneales de anfibios provenientes de egagrópilas de Tyto furcata, Megascops choliba y Athene cunicularia.

\begin{tabular}{|c|c|c|c|c|c|c|c|}
\hline Depredador & Especie presa & Elemento & $\%$ Cat 0 & $\%$ Cat 1 & $\%$ Cat 2 & $\%$ Cat 3 & $\%$ Cat 4 \\
\hline \multirow{4}{*}{ Tyto furcata } & \multirow{4}{*}{ Leptodactylus sp. } & Húmero & 100 & 0 & 0 & 0 & 0 \\
\hline & & Radio-ulna & 100 & 0 & 0 & 0 & 0 \\
\hline & & Fémur & 68,96 & 31,04 & 0 & 0 & 0 \\
\hline & & Tibio-fíbula & 52,78 & 47,22 & 0 & 0 & 0 \\
\hline \multirow{4}{*}{ Megascops choliba } & \multirow{4}{*}{ Rhinella arenarum } & Húmero & 75 & 12,5 & 12,5 & 0 & 0 \\
\hline & & Radio-ulna & 100 & 0 & 0 & 0 & 0 \\
\hline & & Fémur & 64,28 & 28,57 & 7,15 & 0 & 0 \\
\hline & & Tibio-fíbula & 16,66 & 83,34 & 0 & 0 & 0 \\
\hline \multirow{4}{*}{$\begin{array}{l}\text { Athene cunicularia } \\
\text { (SG) }\end{array}$} & \multirow{4}{*}{ Leptodactylus sp. } & Húmero & 55 & 30 & 5 & 5 & 5 \\
\hline & & Radio-ulna & 56,2 & 43,8 & 0 & 0 & 0 \\
\hline & & Fémur & 11,1 & 66,6 & 22,3 & 0 & 0 \\
\hline & & Tibio-fíbula & 0 & 87,5 & 12,5 & 0 & 0 \\
\hline \multirow{4}{*}{$\begin{array}{l}\text { Athene cunicularia } \\
(V R)\end{array}$} & \multirow{4}{*}{ Rhinella arenarum } & Húmero & 16,67 & 16,67 & 16,67 & 33,33 & 16,67 \\
\hline & & Radio-ulna & 16,67 & 66,67 & 16,67 & 0 & 0 \\
\hline & & Fémur & 0 & 100 & 0 & 0 & 0 \\
\hline & & Tibio-fíbula & 0 & 0 & 85,71 & 14,29 & 0 \\
\hline
\end{tabular}




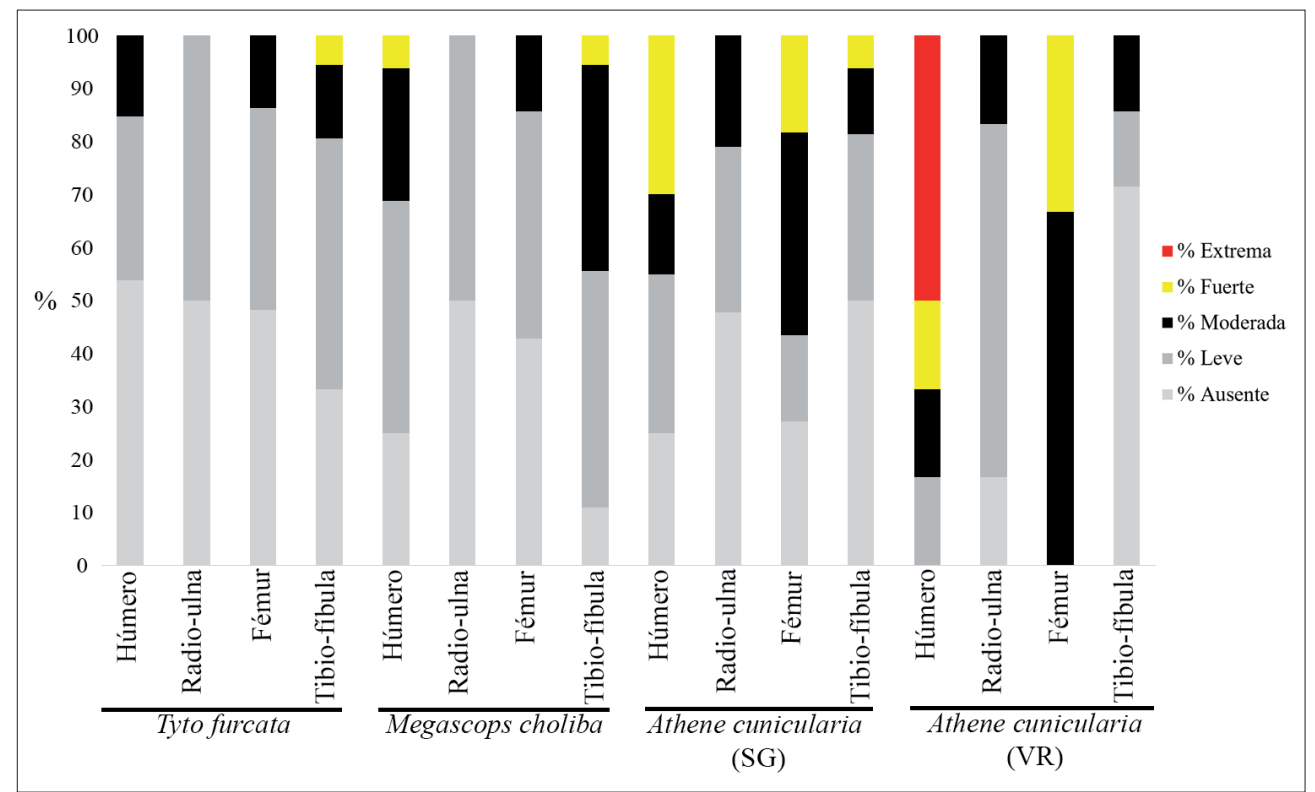

Figura 3. Proporción y categorías de digestión en elementos postcraneales provenientes de egagrópilas de Tyto furcata, Megascops choliba y Athene cunicularia. Abreviaturas: Salinas Grandes (SG); Villa Retiro (VR).

atributo, se registraron daños de tipo fuerte en muestras de A. cunicularia (ca. 10-27\%), mientras que, en conjuntos de $T$. furcata y $M$. choliba, se asociaron únicamente con alteraciones leves. El adelgazamiento fue el atributo observado en menor medida sobre las muestras, a tal punto que los índices no superaron el $7 \%$ en ninguno de los casos estudiados, ubicándose en la categoría leve de modificación. Por otra parte, el colapso se registró en un $5 \%$ de los huesos digeridos por T. furcata y proporciones similares entre muestras de $M$. choliba y A. cunicularia (ca. 12-16\%). Para esta última, se reveló aproximadamente un $6 \%$ con alteraciones de tipo fuertes. Finalmente, se registraron grados leves de descamación sobre los extremos de diáfisis de huesos largos, aunque este atributo se destacó mayormente en muestras de A. cunicularia de VR (22\%) por sobre el resto de las rapaces ( $c a .8-13 \%)$, incluyendo su análogo de SG.

\section{DISCUSIÓN}

Las observaciones realizadas sobre huesos de anfibios permitieron elaborar patrones preliminares sobre los grados de modificación de los elementos anatómicos por parte de distintas aves rapaces estrigiformes. En primera instancia, se observaron promedios de abundancia relativa de partes esqueletales que superaron el $50 \%$ en todos los casos analizados, lo que indicó una buena representatividad de los elementos anatómicos. En concordancia, los valores obtenidos por Montalvo \& Tejerina (2009) en huesos producidos por A. cunicularia, fueron equivalentes a los revelados aquí (51,74\%). En este trabajo, los autores destacaron una mayor representación de húmeros, escápulas, radio-ulnas, iliones y mandíbulas (ver Fig. 2), mientras que, en las muestras provenientes de SG y VR, se destacaron mandíbulas, húmeros y fémures. En huesos digeridos por T. furcata como por M. choliba, se registró mayor representatividad de tibio-fíbulas, húmeros, fémures e iliones que los conjuntos de $A$. cunicularia.

Los índices de representatividad calculados indicaron para $T$. furcata, M. choliba y A. cunicularia (SG) una mejor representación del postcráneo sobre elementos del cráneo, con particularidad en los miembros posteriores. Las proporciones fueron similares a las descritas por Quintana (2015) para muestras de T. furcata. En correlato, Montalvo \& Tejerina (2009) observaron patrones similares, aunque con mejor representación de los miembros anteriores por sobre los posteriores.

La relación entre elementos distales y proximales de los miembros indicó proporciones más equilibradas en las muestras de $T$. furcata, una mejor representación de los primeros por sobre los segundos en muestras de $A$. cunicularia de SG y M. choliba, y una leve tendencia a favor de elementos proximales en muestras de $A$. cunicularia de VR. Esto puede estar vinculado a la forma en que los predadores desmiembran y consumen sus presas y al aporte de carne de las mismas. Al respecto, las tendencias observadas en $A$. cunicularia (SG) y $M$. choliba indicarían mayor consumo de zonas del cuerpo con menor contenido cárnico o la mayor pérdida de elementos 


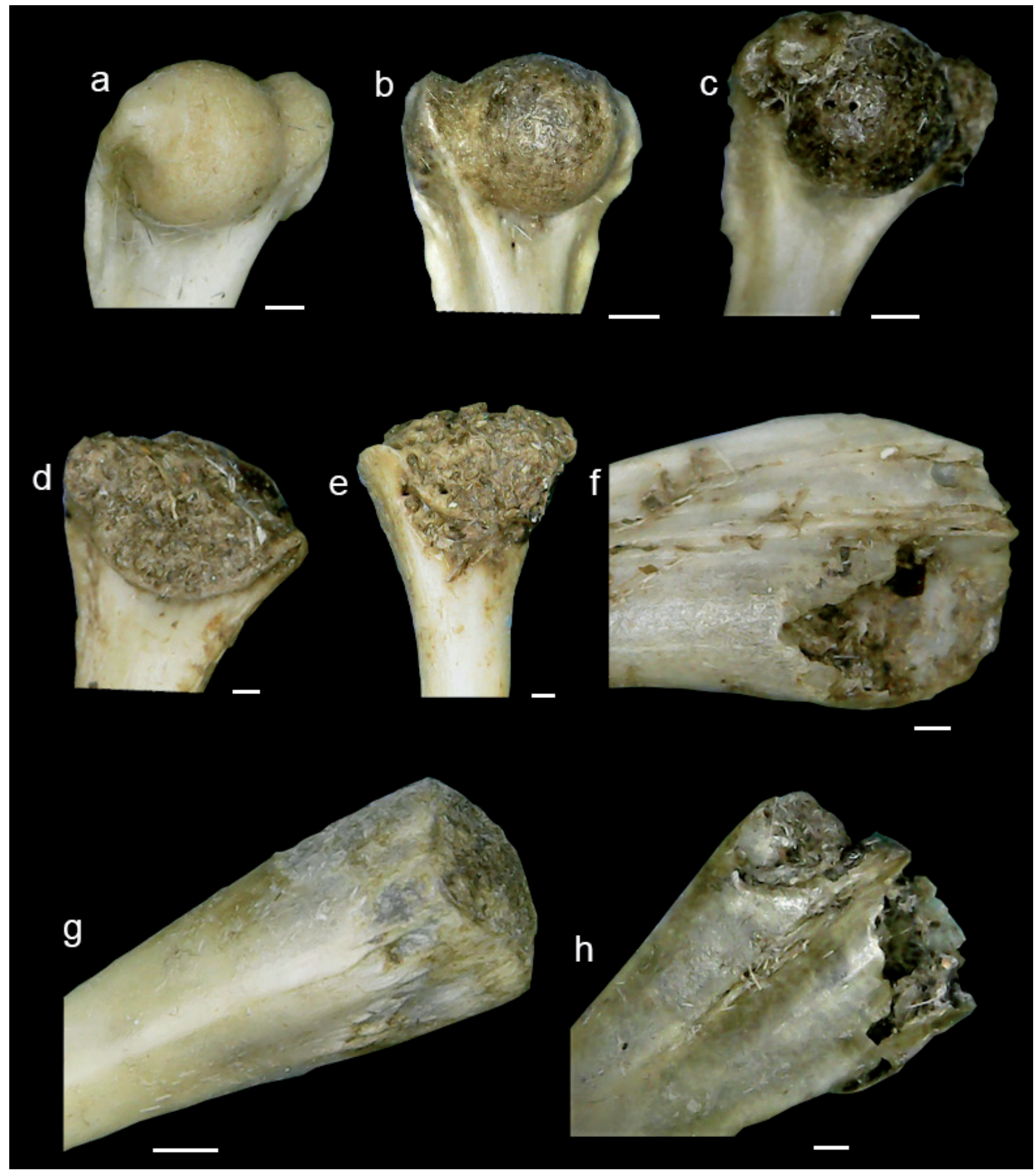

Figura 4. Modificaciones por digestión y fractura registradas en huesos de anfibios contenidos en egagrópilas de Tyto furcata, Megascops choliba y Athene cunicularia. a-e, Distintos grados de modificación por digestión sobre húmeros proximales. f, Tibio-fíbula distal con astillamiento, redondeamiento y colapso. $\mathbf{g}$, Diáfisis de fémur con descamación. $\mathbf{h}$, Distal de radio-ulna con redondeamiento y adelgazamiento. Escala $1 \mathrm{~mm}$. 


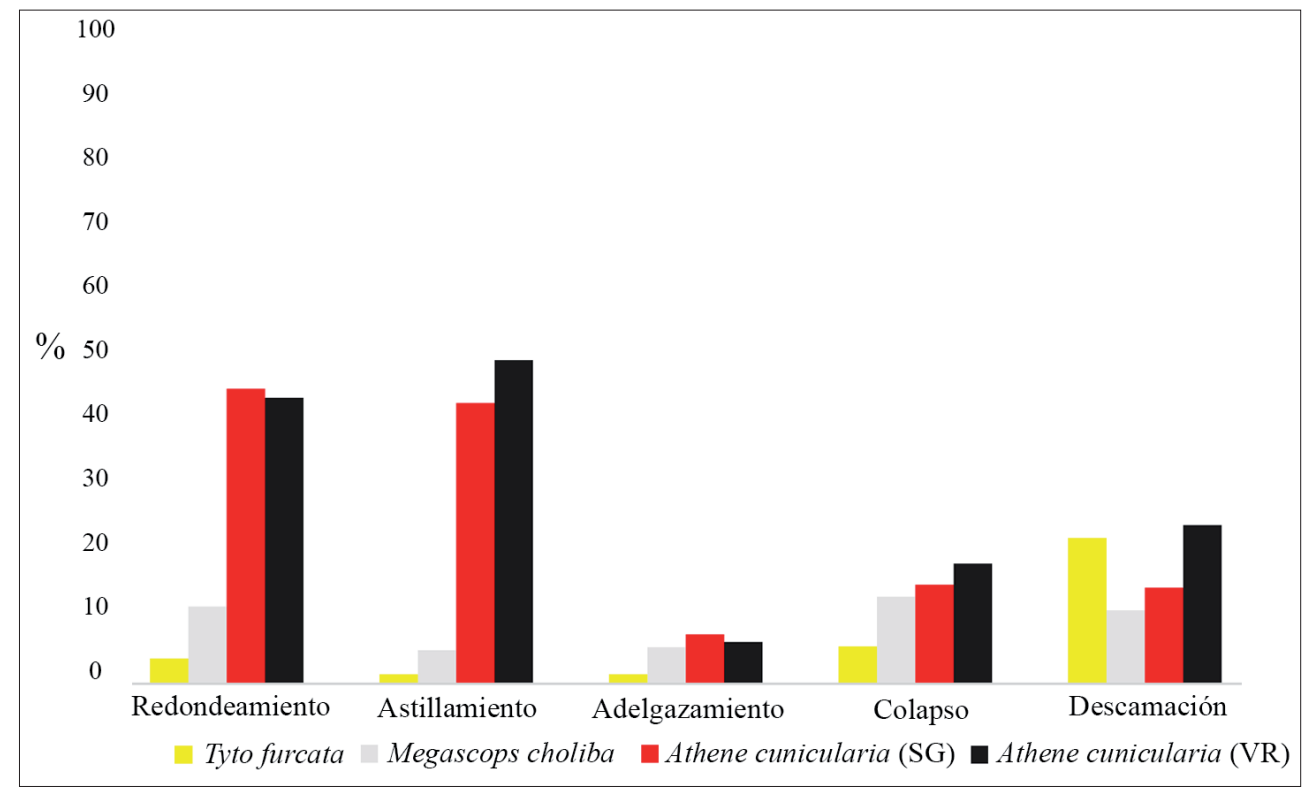

Figura 5. Histograma de porcentajes de atributos tafonómicos en huesos de anfibios contenidos en egagrópilas de Tyto furcata, Megascops choliba y Athene cunicularia. Abreviaturas: Salinas Grandes (SG); Villa Retiro (VR).

proximales durante los procesos de ingestión y posterior digestión (e.g., Raczysnki \& Ruprecht, 1974).

La fragmentación de los elementos del postcráneo sugirió una marcada diferencia entre los restos digeridos por T. furcata sobre aquellos acumulados por $M$. choliba y A. cunicularia (ambas localidades). En concordancia, en estudios llevados a cabo por Pinto-Llona \& Andrews (1999) y Rey \& Sanchiz (2005) sobre restos producidos por $T$. $a l b a$, se observaron patrones similares en comparación con otras estrigiformes y mamíferos carnívoros. Sin embargo, estos autores recuperaron prácticamente la totalidad de los elementos completos mientras que, en las muestras evaluadas en este trabajo, fémures y tibio-fíbulas presentaron algún grado de fractura en las epífisis.

Por otro lado, los huesos fracturados por M. choliba mostraron mayor grado de alteración que los evaluados en T. furcata, sobre todo en tibio-fíbulas, húmeros y fémures.

Las fracturas registradas sobre los huesos producidos por A. cunicularia indicaron elevados porcentajes de modificación en contraposición con lo observado sobre otros conjuntos. Sobre este atributo, Montalvo \& Tejerina (2009) registraron mayor porcentaje de fracturas (cercanos a $80 \%$ ) a los revelados en las muestras de SG, y una mayor similitud con los conjuntos de VR.

$\mathrm{Al}$ igual que en esta última localidad, en los conjuntos estudiados por estos autores se registraron abundantes restos de Rhinella arenarum, un anfibio que puede llegar a medir hasta $150 \mathrm{~mm}$. En cambio, los restos producidos por A. cunicularia (SG), si bien no se logró determinar su identidad específica, indicaron la presencia de ítems presa de tamaño corporal más pequeño. Esto podría explicar, en primera instancia, la relación entre los grados de fractura de elementos anatómicos entre los conjuntos de VR y los evaluados por Montalvo \& Tejerina (2009).

Las diferencias en los porcentajes y grados de fractura posiblemente se vinculen a que T. furcata, con mayor frecuencia, traga sus presas enteras, lo que generaría menor fracturación sobre los miembros. Mientras que, rapaces como A. cunicularia manipulan, en mayor medida, su alimento antes de ingerirlo, lo que generaría más alteraciones sobre los elementos anatómicos (Montalvo \& Tejerina, 2009).

Un aspecto interesante lo constituye la relación entre las fracturas y la abundancia de partes esqueletales. En todos los casos, incluso en aquellos en donde las roturas fueron más elevadas (A. cunicularia), se registraron promedios altos de abundancia relativa de partes esqueletales. Al respecto, se podría pensar que mayor fracturación no necesariamente implican mayor pérdida de elementos anatómicos.

Las modificaciones y atributos causados por acción digestiva avalaron las observaciones previas que caracterizaron a $T$. alba como un modificador leve. Las evaluaciones realizadas por Pinto-Llona \& Andrews (1999) y Quintana (2015) sobre huesos contenidos en egagrópilas de Tyto alba y T. furcata, respectivamente, indicaron valores cercanos al $20 \%$ y $7 \%$, respectivamente. Sin embargo, si bien las modificaciones registradas en este trabajo fueron principalmente leves, las proporciones obtenidas en el conjunto procedente de SG indicaron valores cercanos al $50 \%$ de elementos con trazas de ácidos digestivos.

Esto es interesante, debido a que también se han detectado diferencias en los patrones de fractura y digestión en huesos de micromamíferos presa entre $T$. furcata de 
América y T. alba de Europa y África (Andrews, 1990; López, 2020).

Este no es un hecho menor, dado que la digestión es la variable más confiable a la hora de evaluar la presencia de agentes acumuladores y productores en distintos contextos arqueológicos y paleontológicos, a causa de que otros patrones (e.g., representatividad y fractura) pueden verse sobrestimados por procesos postdepositacionales, como el pisoteo (Andrews, 1990; Fernández et al., 2017).

Las alteraciones por digestión en restos acumulados por $M$. choliba evidenciaron similitud en cuanto a los porcentajes para muestras producidas por A. cunicularia, aunque los grados de alteración fueron principalmente leves.

Por otra parte, A. cunicularia indicó elevadas proporciones de modificación. En consistencia con otros antecedentes, los efectos de la digestión abarcaron todas las categorías.

Finalmente, los resultados alcanzados sobre fractura y digestión fueron comparados con los obtenidos por PintoLlona \& Andrews (1999) en donde se incluyeron aves rapaces y mamíferos carnívoros. Acorde con los resultados de estos autores, la Figura 6 muestra que, en general, los mamíferos carnívoros producen una mayor fracturación que las aves rapaces. Mientras que estas últimas exhiben mayor modificación por digestión. Un elemento particular lo constituyen los ensambles producidos por $A$. cunicularia en donde se registraron porcentajes elevados para ambos atributos.

\section{CONCLUSIONES}

Este trabajo constituye uno de los primeros estudios tafonómicos comparativos de anfibios acumulados por distintos agentes productores sudamericanos.

Si bien este resulta un estudio preliminar, las evaluaciones derivadas de los resultados permitieron obtener tendencias sobre los patrones de modificación que generan distintas aves rapaces estrigiformes sobre los restos de anfibios presa en el centro de Argentina. Estos primeros datos se constituyeron como un insumo de utilidad a la hora de evaluar la presencia de restos de anfibios en contextos arqueológicos y paleontológicos, y su posible vinculación con determinados agentes productores.

Los datos revelados aquí indican que las observaciones sobre huesos digeridos por T. furcata ubican a esta rapaz en la categoría leve de modificación por fractura y digestión, al igual que en restos de pequeños mamíferos. Sin embargo, los porcentajes obtenidos en este estudio indican mayor modificación que los alcanzados en contextos europeos y africanos.

Acorde a los parámetros observados sobre las alteraciones en elementos producidos por M. choliba, estos indicarían que los porcentuales y grados de fractura y digestión sobre los huesos son superiores a los registrados para $T$. furcata. Los atributos tafonómicos fueron categorizados como leves a moderados.

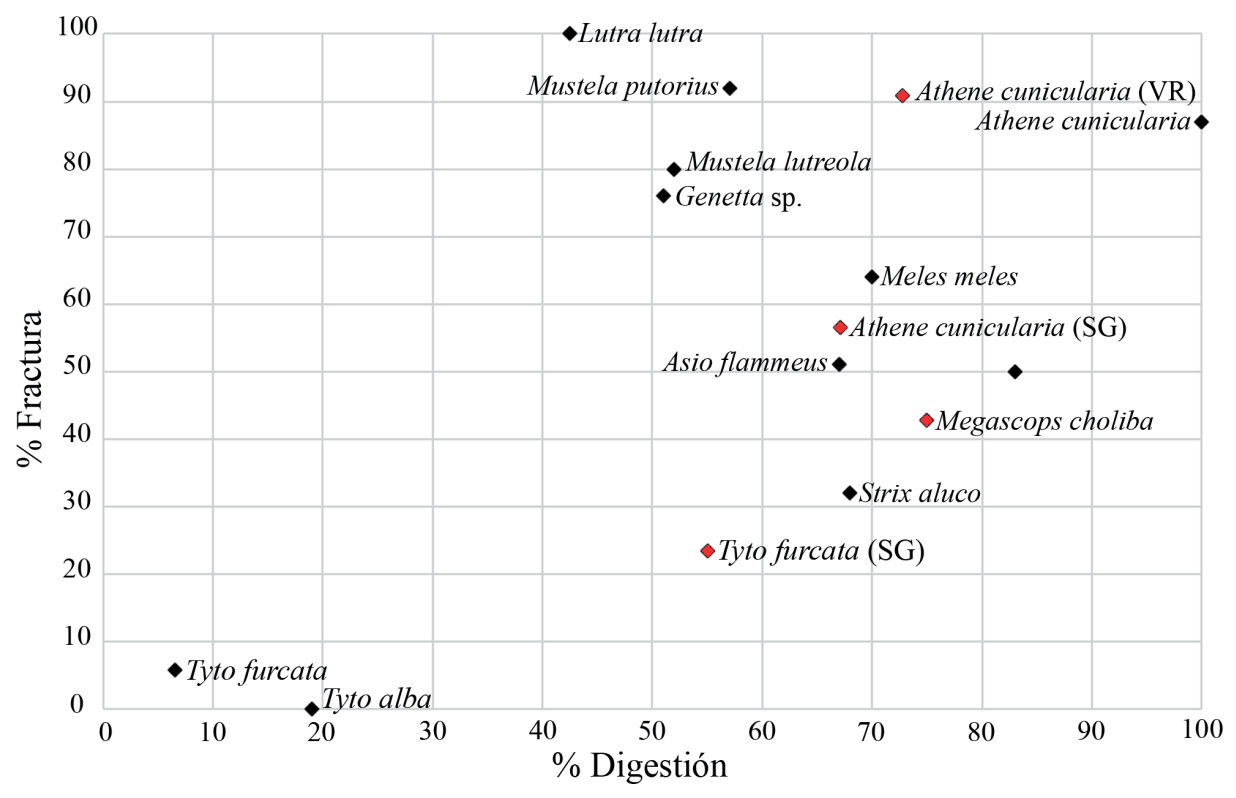

Figura 6. Gráfico bivariado sobre fractura y digestión ocasionada por distintos depredadores. Asio flammeus, Bubo bubo, Strix aluco, Tyto alba, Lutra lutra, Mustela putorius, Mustela lutreola, Genetta sp. (Pinto-Llona \& Andrews, 1999); Athene cunicularia (Montalvo \& Tejerina, 2009); Tyto furcata (Quintana, 2015); Tyto furcata (Salinas Grandes), Athene cunicularia (Salinas Grandes), Athene cunicularia (Villa Retiro), Megascops choliba (este trabajo). 
Las evaluaciones de restos producidos por M. choliba constituyen el primer antecedente de un estudio tafonómico en huesos de anfibios para esta rapaz.

Finalmente, las evaluaciones sobre restos contenidos en egagrópilas de $A$. cunicularía indicaron grados de alteraciones principalmente moderadas, con altos porcentajes de redondeamiento y astillamiento. Esto confirmaría su ubicación en la categoría moderada de modificación. La ampliación de muestreos y análisis permitirán comparar estas observaciones.

\section{AGRADECIMIENTOS}

La Dra. Yolanda Fernández-Jalvo y la Dra. Paloma Sevilla fueron responsables de la mejoría en la calidad de este manuscrito. Agradezco al Dr. Julio Aguirre y Carlos Martínez-Pérez por considerar y mejorar la calidad de este trabajo. El Dr. Fernando Fernández realizó valiosos aportes sobre el manuscrito original. Oscar Vives brindó asistencia técnica. Agradezco a quienes apoyan desde la formación y soporte financiero: PICT 2016-0264 (Roxana Cattáneo, Directora) PIP CONICET 11220130100137CO (Andrés Izeta, Director).

\section{BIBLIOGRAFÍA}

Alcaraz, A.P. 2017. La fauna menor de sitios arqueológicos del curso inferior del Colorado (provincia de Buenos Aires): aspectos tafonómicos y subsistencia de cazadores recolectores durante el Holoceno medio y tardio. Tesis doctoral, Facultad de Ciencias Sociales, Universidad Nacional del Centro de la Provincia de Buenos Aires (unpublished).

Andrews, P. 1990. Owls, Caves and Fossils. Predation, Preservation, and Accumulation of Small Mammal Bones in Caves, with the Analysis of the Pleistocene Cave Faunas from Westbury-sub-mendip, Somerset. UK. Natural History Museum Publications, London.

Bellocq, I. 2000. A review of the tropic ecology if the Barn Owl in Argentina. Journal of Raptor Research, 34, 108-119.

Bisbal-Chinesta, J.F., Cardona-Bañuls, S., FernándezGarcía, M., Caseres, I., Blain, H. A. \& Vergés, J.M. 2020. Elucidating anuran accumulations: massive taphocenosis of tree frog Hyla from the Chalcolithic of El Mirador cave (Sierra de Atapuerca, Spain). Journal of Archaeological Science: Reports, 30, 102277, doi: 10.1016/j.jasrep.2020.102277.

Bochenski, Z.T., Tomek, T., Tornberg, R. \& Wertz, K. 2009. Distinguishing nonhuman predation on birds: pattern of damage done by the white-tailed Eagle Haliaetus albicilla, with comments on the punctures made by the golden eagle
Aquila chrysaetos. Journal of Archaeological Science, 36, 122-129.

Blain, H. A., Bailon, S. \& Cuenca-Bescós, G. 2008. The Early-Middle Pleistocene palaeoenvironmental change based on the squamate reptile and amphibian proxy at the Gran Dolina site, Atapuerca, Spain. Palaeogeography. Palaeoclimatology. Palaeoecology, 261, 177-192, doi: 10.1016/j.palaeo.2008.01.015.

Cabido, M., Zeballos, S.R., Zak, M., Carranza, M., Giorgis, M., Cantero, J. \& Acosta, A. 2018. Native woody vegetation in central Argentina: Classification of Chaco and Espinal forests. Applied Vegetation Science, 21, 298-311, doi: 10.1111 avsc. 12369 .

Delgado, C.A. 2007. La dieta del Currucutú Megascops choliba (Strigidae) en la ciudad de Medellin, Colombia. Boletin SAO, 17, 111-114.

del Hoyo, J., Elliot, A.J. \& Sargatal (eds.). 1999. Handbook of the birds of the World. Barn owls to hummingbirds. Vol 5. Lynx Edicions, Barcelona.

Fernández-Jalvo, Y. \& Andrews, P. 1992. Small mammal taphonomy of Gran Dolina, Atapuerca (Burgos), Spain. Journal of Archaeological Science, 19(4), 407-428, doi: 10.1016/0305-4403(92)90058-B.

Fernández, F.J., Moreira, G., Ferraro, D. \& De Santis, L. 2009. Presas consumidas por la lechuza de campanario (Tyto alba) en la localidad de Olavarría, Buenos Aires: un caso de elevada batracofagia. Nuestras Aves, 54, 20-21.

Fernández, F.J., Del Papa, L., Moreira, G., Prates, L. \& De Santis, L. 2011. Small mammal remains recovered from two archaeological sites in the middle and lower Negro River valley (Late Holocene, Argentina). Taphonomic issues and paleoenvironmental implications. Quaternary International, 245, 136-147, doi: 10.1016/j.quaint.2010.12.027.

Fernández, F.J., Teta, P., Barberena, R. \& Pardiñas, U.F.J. 2012. Small mammal remains from Cueva Huenul 1, northern Patagonia, Argentina: Taphonomy and paleoenvironments since the Late Pleistocene. Quaternary International, 278, $22-31$.

Fernández, F.J., Montalvo, C.I., Fernández-Jalvo, Y., Andrews, P. \& López, J.M. 2017. A reevaluation of the taphonomic methodology for the study of small mammal fossil assemblages of South America. Quaternary Science Reviews, 155, 37-49, doi: 10.1016/j.quascirev.2016.11.005.

Furts, P.T. 1994. Alucinógenos y cultura. Fondo de Cultura Económica, México.

Frontini, R. \& Escosteguy, P. 2015. El rol de los pequeños animales en los estudios arqueofaunísticos de Argentina. Archaeofauna, 24, 67-85.

Hilty, S.L. \& Brown, L. 1986. A guide to the Birds of Colombia. Princeton University Press, New Jersey.

Hüster, H. 2004. Fischfang und Kleintierbeute. Ergebnisse der Untersuchung aus den Schlämmproben. In: Die jungsteinzeitliche Seeufersiedlung Arbon-Bleiche 3 (eds. Jacomet, S., Leuzinger, U. \& Schibler, J.). Thurgau, Umwelt und Wirtschaft, 253-276.

Kyselý, R. 2008. Frogs as a part of the Eneolithic diet. Archaeozoological records from the Czech Republic (Kutná Hora-Denemark site, Rivnác Culture). Journal 
of Archaeological Science, 35, 143-157, doi: 10.1016/j. jas.2007.02.016.

König, C. \& Weick, F. 2010. Owls of the world. A\&C Black Publishers Ltd, London.

López, J.M. 2020. Actualistic taphonomy of barn owl pellet-derived small mammal bone accumulations in arid environments of South America. Journal of Quaternary Science, 35, 1057-1069. doi: $10.1002 /$ jqs.3251.

Mignino, J., Izeta, A. \& Cattáneo, R. 2018. Modern and archaeological owl pellets as paleoenvironmental and taphonomic markers in human occupation contexts in the Ongamira Valley, Córdoba, Argentina. Journal of Archaeological Science: Reports, 18, 65-77. doi: 10.1016/j. jasrep.2017.12.054.

Montalvo, C.I. \& Fernández, F.J. 2019. Review of the actualistic taphonomy of small mammals ingested by South American predators. Its importance in the interpretation of the fossil record. Publicación electrónica de la Asociación Paleontológica Argentina, 19(1), 18-46, doi: 10.5710/ PEAPA.11.03.2019.275.

Montalvo, C.I. \& Tejerina, P. 2009. Análisis tafonómico de los huesos de anfibios y roedores depredados por Athene cunicularia (Strigiformes, Strigidae) en La Pampa, Argentina. In: Mamul mapu: pasado y presente desde la arqueología pampeana (eds. Berón, M., Luna, L., Bonomo, M., Montalvo, C. I., Aranda, C. \& Carrera Aizpitarte, M.). Editorial Libros del Espinillo, Buenos Aires, 323-334.

Montalvo, C., Fernández F.J., Tomassini, R., Mignino, J., Kin, M. \& Santillan, M.A. 2020. Spatial and temporal taphonomic study of bone accumulations of the burrowing owl (Athene cunicularia) in central Argentina. Journal of Archaeological Science: Reports, 30, 102197, doi: 10.1016/j.jasrep.2020.102197.

Muzzopappa, P., Martinelli, A., Garderes, J.P. \& Rougier, G. 2020. Exceptional avian pellet from the paleocene of Patagonia and description of its content: a new species of calyptocephalellid (Neobatrachia) anuran. Papers in Palaeontology, 1-14, doi: 10.1002/spp2.1333.

Pardiñas, U.F.J. 1999. Tafonomía de microvertebrados en yacimientos arqueológicos de Patagonia (Argentina). Arqueología, 9, 265-340.
Pokines, J. \& Kerbis, J. 1997. Barn Owl (Tyto alba) taphonomy in the Negev Desert, Israel. Israel Journal of Zoology, 43, 19-27.

Pinto-Llona, A.C. \& Andrews, P. 1999. Amphibian taphonomy and its application to the fossil record of Dolina (middle Pleistocene, Atapuerca, Spain). Palaeogeography, Palaeoclimatology, Palaeoecology, 149, 411-429.

Quintana, C. 2015. Tafonomía de contenidos dispersos de egagrópilas de Tyto alba en un ambiente serrano. Historia Natural, Tercera Serie, 5(1), 29-47.

Raczynski, J. \& Ruprecht, A.L. 1974. The effect of digestion on the osteological composition of owl pellets. Acta Ornithologica, 14, 25-38.

Rey, J.M. \& Sanchiz, B. 2005. Differential anuran bone preservation in a taphocenotic sample of Barn owl pellets. Munibe (Antropologia-Arkeologia), 57, 505-509.

Robledo, A. 2019. Arqueología en el valle de Ongamira (Deptos. de Ischilín y Totoral, Córdoba, Argentina). Paisajes y lugares de sociedades cazadoras recolectoras holocénicas. Tesis doctoral, Facultad de Filosofía y Humanidades, Universidad Nacional de Córdoba (unpublished).

Schultes, R.E. \& Hofmann, A. 2000. Plantas de los dioses. Orígenes del uso de los alucinógenos. Fondo de Cultura Económica, México.

Stoessel, L., Bogan, S., Martínez, G. \& Agnolin, F.L. 2008. Implicaciones paleoambientales de la presencia del género Ceratophrys (Anura, Ceratophryinae) en contextos arqueológicos de la transición pampeano-patagónica en el Holoceno Tardío (curso inferior del Río Colorado, Argentina). Magallania (Punta Arenas), 36(2), 195-203, doi:10.4067/S0718-22442008000200015.

Stoetzel, E., Denys, C., Bailon, S., El Hajraoui, M.A. \& Nespoulet, R. 2012. Taphonomic analysis of amphibian and squamate remains from El Harhoura 2 (RabatTémara, Morocco): contributions to palaeoecological and archaeological interpretations. International Journal of Osteoarchaeology, 22, 616-635, doi: 10.1002/oa.1275.

Uva, V., Päckert, M., Cibois, A., Fumagalli, L. \& Roulin, A. 2018. Comprehensive molecular phylogeny of barn owls and relatives (Family: Tytonidae), and theirsix major Pleistocene radiations. Molecular Phylogenetics and Evolution, 125, 127-137. 